\title{
AN OPTICAL STUDY OF THE POSSIBLE PROTO-STARBURST GALAXY VII ZW 31
}

\author{
S. Djorgovski, a) R. R. DE Carvalho, and D. J. Thompson \\ Palomar Observatory, Division of Physics, Mathematics, and Astronomy, California Institute of Technology, Pasadena, California 91125 \\ Received 15 November 1989; revised 19 January 1990
}

\begin{abstract}
We present an optical imaging and spectroscopic study of an ultraluminous, gas-rich Infrared Astronomical Satellite (IRAS) galaxy, VII Zw 31. Sage and Solomon proposed earlier that this object may be interpreted as a protogalactic disk at low redshift, on account of its large mass fraction of molecular gas. Our data suggest that the object is a merger-induced starburst, and in that respect similar to many other ultraluminous $I R A S$ galaxies. Asymmetries and distortions are seen in the visible light, and a prominent dust lane is detected near the nucleus. The spectra show strong, low-ionization line emission, consistent with photoionization by young stars, and substantial internal extinction. Absorption spectra suggest a presence of both young and old stellar populations. We interpret the object as an old galaxy, rejuvenated by a recent starburst. Since the large molecular gas content ( $\sim 50 \%$ of the total mass) is the main distinguishing characteristic of this object, the starburst must be just in its initial stages. VII Zw 31 may thus be a low-redshift analog of at least some protogalaxies at large redshifts.
\end{abstract}

\section{INTRODUCTION}

Two recent lines of development in extragalactic astronomy contributed to the broadening of our understanding of galaxy formation. First is the realization that galaxy formation is probably an ongoing series of processes continuing to the present epoch, rather than a single cataclysmic event which happened at a large redshift. Second is the discovery of the multitude of luminous starburst galaxies by the IRAS satellite, which are often inconspicuous optically, but contribute substantially to the net star formation (and hence the energy release) in the local universe. The two strands may be connected in the remarkable galaxy VII Zw 31.

This object was first cataloged by Zwicky (1971), with a terse remark: "Blue, patchy spherical compact, $m_{p}=15.8$." It was identified as an ultraluminous $\left(L_{\mathrm{FIR}} \sim 10^{12} L_{\odot}\right)$ IRAS galaxy by Fairclough (1986). Then Sage and Solomon (1987) discovered that the object has the highest CO $\lambda 2.6$ $\mathrm{mm}(J=1 \rightarrow 0)$ luminosity known until then, $L_{\mathrm{CO}}$ $\simeq 1.1 \times 10^{10} \quad \mathrm{~K} \mathrm{~km} \mathrm{~s}^{-1} \mathrm{pc}^{2}$ (assuming $H_{0}=75$ $\mathrm{km} \mathrm{s}^{-1} \mathrm{Mpc}^{-1}$ ). This corresponds to mass in molecular hydrogen of $\mathscr{M}_{\mathrm{H}_{2}} \sim 5 \times 10^{10} \mathscr{M}_{\odot}$, which is about a half of the total dynamical mass inferred for the galaxy. Both $L_{\mathrm{CO}}$ and $\mathscr{M}_{\mathbf{H}_{2}}$ are higher for VII Zw 31 than for Arp 220, the prototypical ultraluminous $I R A S$ starburst galaxy. The inferred star-formation rate is $\sim 60 \mathscr{M}_{\odot} \mathrm{yr}^{-1}$, assuming a normal IMF. However, as Sage and Solomon also point out, the most interesting aspect of this discovery may be the high ratio of the $\mathscr{M}_{\mathrm{H}_{2}}$ to the total mass, $\sim 40-70 \%$, depending on the unknown projection effects. This ratio suggests that as ultraluminous as the object may be, it still has to convert most of its mass into stars. Based on that fact, Sage and Solomon proposed that the object is a possible protogalactic disk at a low redshift, or alternatively an extremely gas-rich merger.

The extreme properties of VII Zw 31 and its possible protogalactic nature call for more studies. Further $\mathrm{CO}$ measurements and inconclusive optical imaging of the object were published by Scoville et al. (1989), and Goldsmith and Young (1989); they improved on the original CO measure-

a) Alfred P. Sloan Foundation fellow. ments by Sage and Solomon, but did not change appreciably the conclusions about the extreme properties of this object. Scoville et al. demonstrated that the CO emission originates from a compact central region not larger than a few kpc. Goldsmith and Young also provide upper limits on the molecular $\mathrm{O}_{2}$ emission. Additional information about the nature of this object can be obtained in the optical regime, which served as a motivation for this study.

Throughout this paper we assume $H_{0}=75$ $\mathrm{km} \mathrm{s}^{-1} \mathrm{Mpc}^{-1}$, giving the scale of $1.05 \mathrm{kpc} / \operatorname{arcsec}$ at the source $(z=0.05424)$.

\section{IMAGING AND PHOTOMETRY}

Direct images of VII Zw 31 were obtained at the Palomar 60 in. telescope on the nights of UT 1988 January 20 and 21. The detector was a TI $800 \times 800 \mathrm{CCD}$ with reimaging optics. On January 20, the data were obtained in the $2 \times 2$ binning mode, resulting in the effective pixel scale of 1.23 arcsec; on January 21 , direct $1 \times 1$ binning mode was used, with the resulting pixel scale 0.615 arcsec. The conditions were photometric on both nights, with the seeing $\mathrm{FWHM} \simeq 3$ arcsec on January 20 , and the seeing FWHM $\simeq 2 \operatorname{arcsec}$ on January 21 . Therefore, we use only the January 21 data for the morphological part of this investigation, and the data from both nights for the photometry.

The total of 19 galaxy exposures, ranging from 300 to 500 $\mathrm{s}$, were obtained in the Gunn-Thuan gri bands. The telescope was always moved a few arcseconds between the exposures, in order to combat the low-level systematics. The data were processed using standard techniques. All images were digitally shifted into coincidence, cleaned from the cosmic rays and $\mathrm{CCD}$ artifacts, and the images in corresponding bands were added together.

Multiple exposures of the standard stars BD + $26^{\circ} 2606$, Feige 34, and Feige 67 (Kent 1985) were also obtained, in order to provide the magnitude zero-point calibration. Atmospheric extinction coefficients for Palomar from Dr. A. Porter (private communication) were used. We find the typical zero-point variations between different stars and exposures to be on the level of $\sim 0.01$ mag.

Figure 1 shows a subsection of the digital stack of all images ( gri) from January 21 data. The distorted shape of the 


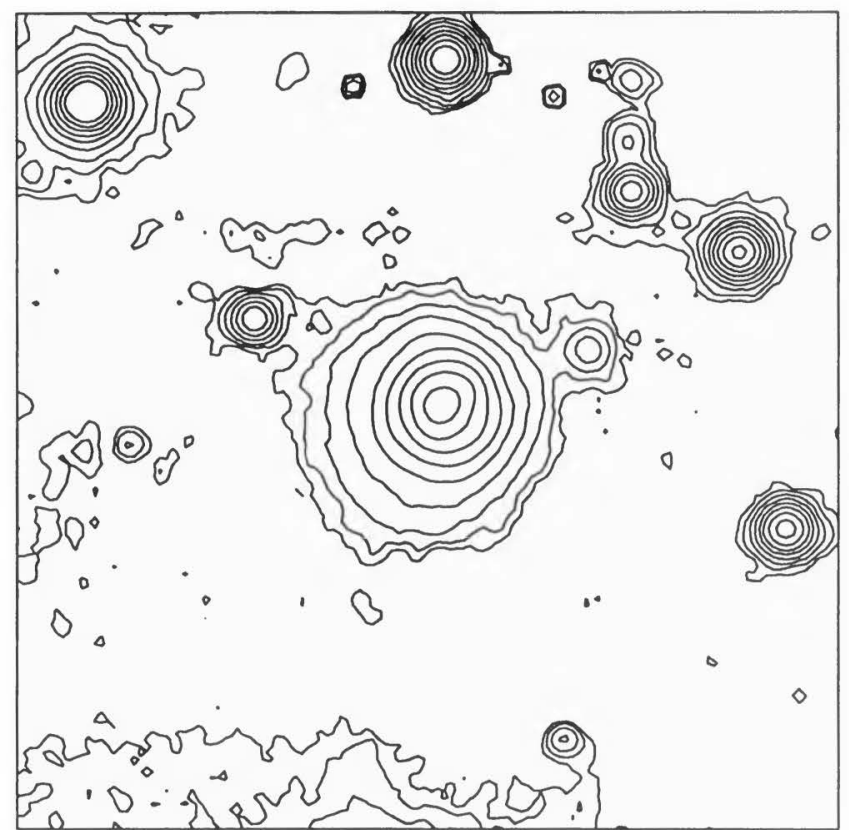

FIG. 1. Subsection of the digital stack of all images ( $g r i)$ from January 21 data. The field shown is 1.31 arcmin square, with north to the top and east to the left. Note the asymmetry of the isophotes.

isophotes is evident, but there is no other morphological evidence at large radii for a tidal interaction or a past merger to the limit of our data. According to Goldsmith and Young (1989), a spectrum taken by Dr. J. Huchra shows that the object to the NE of the galaxy is a foreground star. However, the object to the NW is extended, and may be either a com- panion dwarf galaxy, or an undigested remnant of the possible merged partner.

One can do much better using simple image-processing techniques. A now standard method to search for dust and other features in elliptical galaxies is the subtraction of (or the division by) a model image derived from surface photometry. Model images have elliptical symmetry, and the surface brightness, ellipticity, and major-axis position angle (P.A.) profiles derived from the data, usually with some smoothing. This simple but powerful technique essentially removes the strong radial gradients in galaxy images and reveals any deviations from simple elliptical symmetry. Some examples and earlier references can be found in $\mathrm{Eb}$ neter et al. (1987); a discussion and a comparison of different techniques is given by Djorgovski (1986).

The central part of our stack image from January 21 and the model-subtracted version are shown in Fig. 2. The original image shows just a hint of a distortion, ovoidal, and distorted isophotes near the center, also visible in the contour map published by Goldsmith and Young (1989). The model-subtracted image reveals a much more complex situation. There is a prominent dust lane just SW from the apparent center of the light, which probably partly obscures the true center of the galaxy. The depth of the feature is about $25 \%$ in our images (limited by the seeing). Its relative position is consistent with the compact CO core found by Scoville et al. (1989). The morphology of the residual light is asymmetric and patchy over the whole central region. Essentially the same results are obtained in all three bands and in the stack image, except that the dust lane is more prominent in the bluer bands. The whole picture is consistent with the object being a merger remnant, similar to many other ultraluminous IRAS galaxies.

Surface photometry was performed on the images using (a)

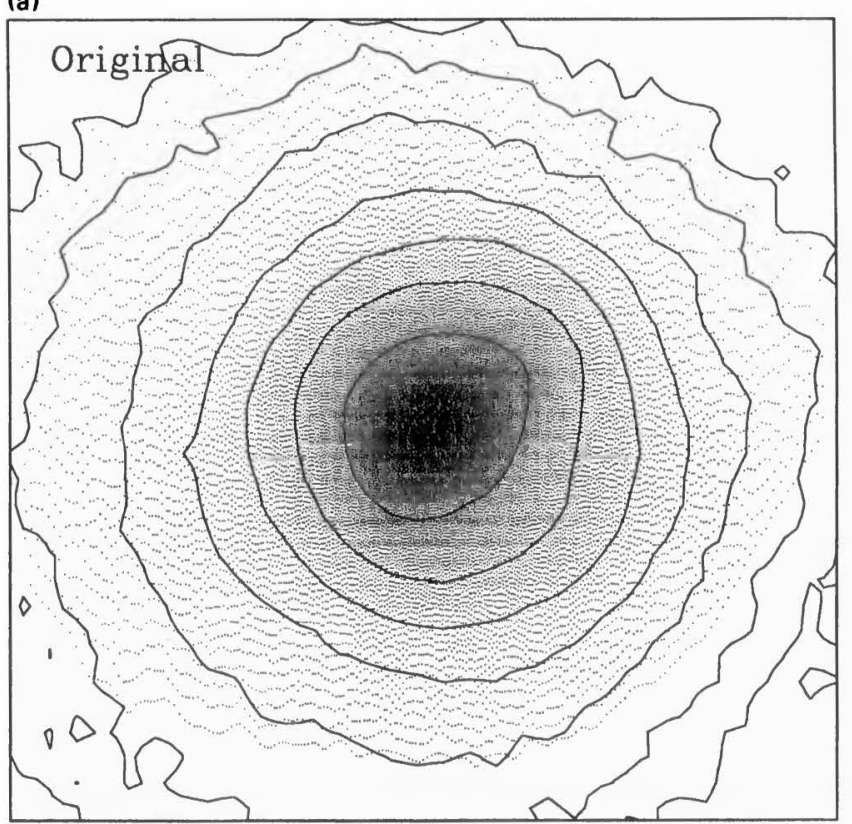

(b)

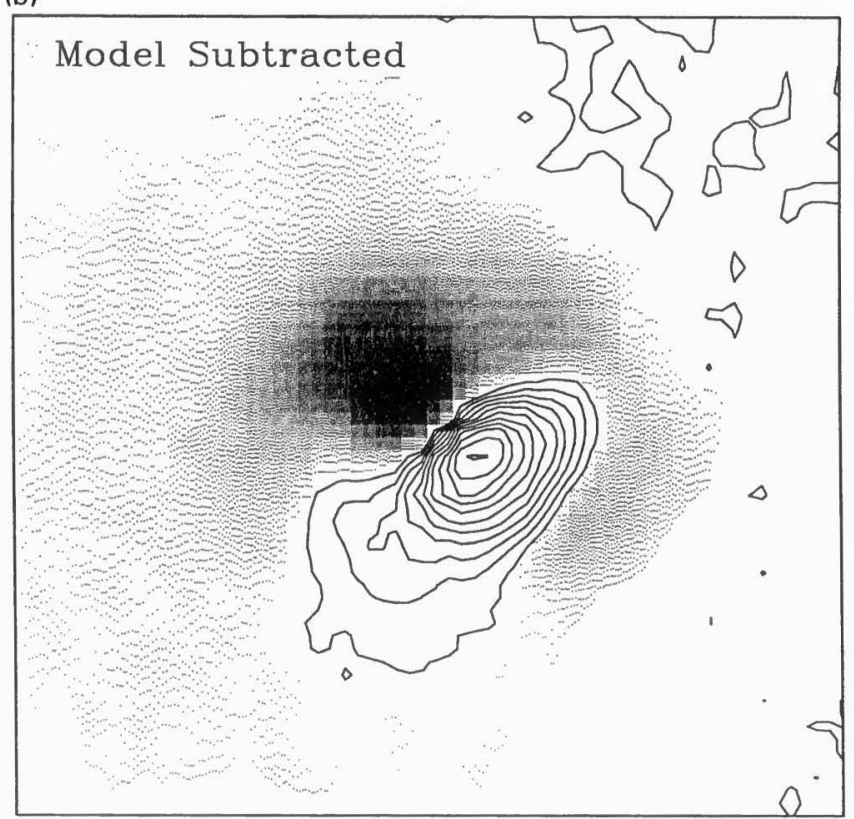

FIG. 2. Central portion of the stack image shown in Fig. 1. The field shown is 19.7 arcsec square. (a) Original image. Both the gray scale and the contours indicate the relative intensity, with the contours spaced by a factor of 2. (b) Same image, but with the elliptically symmetric model subtracted. The positive residuals are shown as the gray scale; note the patchy morphology. The negative residuals (most likely a signature of a dust lane) are indicated by the contours, which are spaced linearly. The depth of the absorption feature is about $25 \%$ in this stack frame. 
both an elliptical isophote fitting routine (as described by Djorgovski 1985), and a circular aperture averaging. Care was taken to use exactly the same galaxy center in evaluating all profiles ( $g, r$ and $i$ separately, and the stack). From the multiple exposures on both nights, we estimate that the net magnitude zero-point uncertainties are about $0.05 \mathrm{mag}$ in each band. The major-axis surface-brightness profiles are shown in Fig. 3. The error bars indicate the internal errors, due to the Poisson noise in the data, and the sky subtraction and flatfielding uncertainties, and do not include the magnitude zero-point errors (they just shift the profiles vertically, and are fairly small). Ellipticity and P.A. profiles for the stack image are shown in Fig. 4; the profiles for the individual bandpasses are essentially identical, within the errors, and the stack image has the highest signal-to-noise. Color profiles were evaluated from the circular aperture photometry, in order to avoid mismatches in ellipticity and P.A. for the individual profiles. Care was taken to use exactly the same galaxy center for all three bands. The color profiles are shown in Fig. 5. We note that the color zero points are uncertain by about $0.1 \mathrm{mag}$, but the shapes of the profiles should be unaffected.

Both surface-brightness and color profiles show a strong reddening towards the center, especially at radii smaller than about 6-8 arcsec. This effect is undoubtedly caused by the dust lane revealed in our images. At radii greater than about 11 arcsec, the surface-brightness profiles are very similar in shape, with the logarithmic slopes $\sim 2$, typical for elliptical galaxies and bulges. Consequently, the color profiles are almost flat in that region. The ellipticity and P. A. profiles show correlated features, which can be easily identified with the apparent distortions in the isophotes.

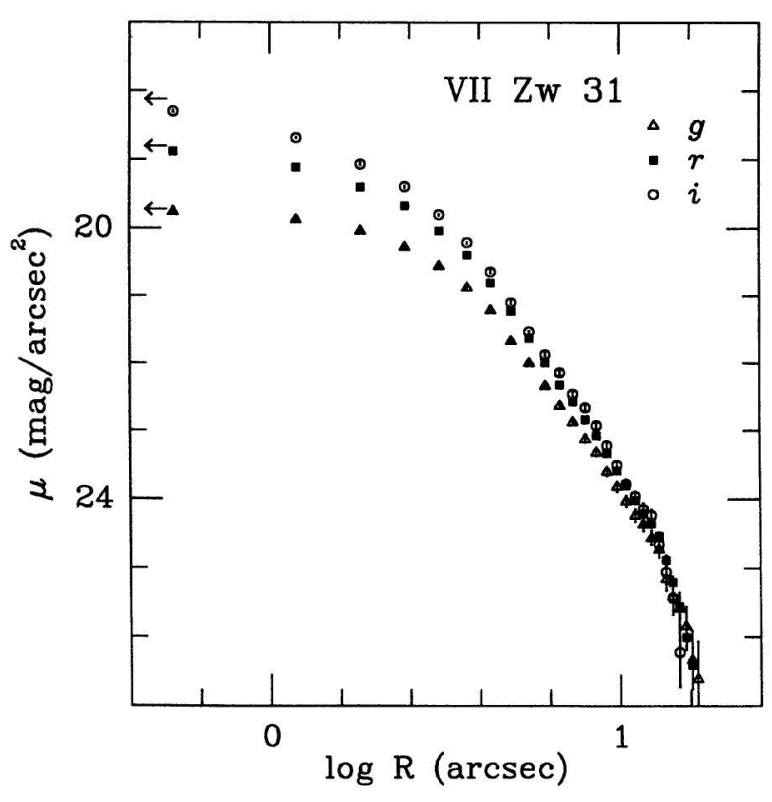

FIG. 3. Major-axis surface-brightness profiles derived from the stacked images from January 21 data. Different bandpasses are encoded with different symbols, as indicated. The error bars indicate the internal errors only; the magnitude zero-point calibration errors are about $0.05 \mathrm{mag}$ in each band. The central values are indicated by the arrows.

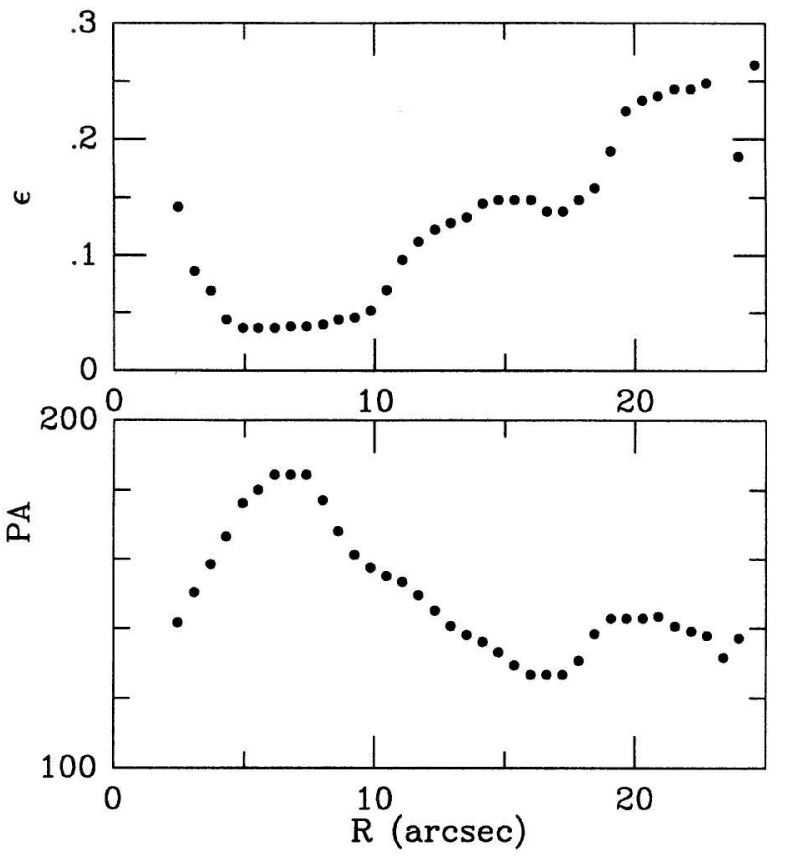

FIG. 4. Ellipticty and major-axis P.A. profiles for the gri stack image. Note the excellent correlation of features seen in both profiles.

The integrated magnitudes of the galaxy are: $g=15.22$, $r=14.76$, and $i=14.75$, with errors of about $0.05 \mathrm{mag}$. The integrated colors are: $(g-r)=0.53$ and $(r-i)=0.24$, with errors of about 0.1 mag. From the maps by Burstein and Heiles (1982), we estimate the foreground Galactic extinction $E_{B-V}=0.09$ in this direction, corresponding to $A_{g}$ $\simeq 0.30, A \simeq 0.23$, and $A_{i} \simeq 0.18$. Correcting the observed magnitudes, and assuming the distance modulus $(m-M) \simeq 36.7$, the optical luminosity of VII $\mathrm{Zw} 31$ is somewhat brighter than that of an $L_{*}$ galaxy. The asympto-

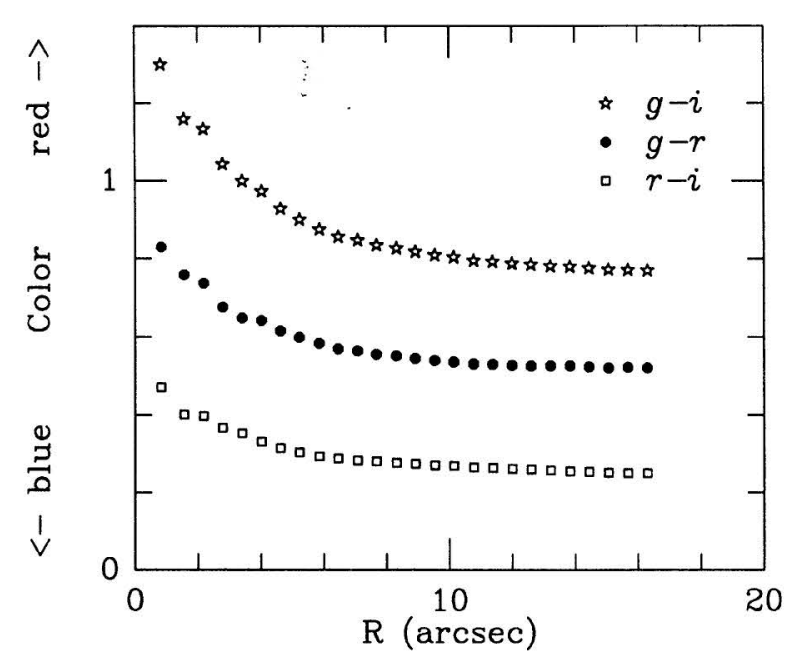

FIG. 5. Color profiles, derived from circular annular apertures. The zero points are uncertain by about $0.1 \mathrm{mag}$. The strong reddening at small radii is most likely caused by the central dust lane. 
tic corrected color $(g-r) \simeq 0.7$ corresponds approximately to the spectral $\mathrm{K}$ type, but it could be affected by an internal diffuse dust component in the object.

A short $K$-band exposure taken at the Palomar 200 in. by S. D. and Dr. Keith Matthews shows only the smooth, resolved core of the galaxy, with no obvious central point source (e.g., a hidden active nucleus).

\section{SPECTROSCOPY}

Long-slit CCD spectra of the object were obtained on the night of UT 1988 January 10, using the Double Spectrograph (Oke and Gunn 1982) mounted in the Cassegrain focus on the Hale 200 in. telescope. The conditions were nonphotometric, with variable transparency, and the seeing was never better than about 2 arcsec (FWHM). The slit width used was 2 arcsec, and the effective spectral resolution (FWHM) was about $10 \AA$. The effective wavelength range covered was about $3700-4300 \AA$ with the blue channel, and about 5000-7500 $\AA$ with the red channel. Two exposures were obtained, of 600 and $1200 \mathrm{~s}$ duration. Exposures of arc lamps were used to obtain the dispersion solutions, giving the wavelength calibration rms of $\sim 1 \AA$. Dome flatfields and exposures of standard stars from Oke and Gunn (1983) were used to correct for the instrument response and to provide a crude flux calibration. The data were reduced using standard procedures.

The fully reduced spectra are shown in Fig. 6, with the prominent absorption and emission features labeled. The spectra are typical of a starburst galaxy. Note the prominence of the Balmer absorption lines, suggesting a relatively young stellar population. However, the lines of $\mathrm{Na}, \mathrm{Mg}$, and $\mathrm{Ca}$ are also very strong, indicating an older stellar component as well. The $\mathrm{H} \beta$ / [O III 5007 ratio suggest a low ionization, typical for an extragalactic $\mathrm{H}$ II region, and the lines show no appreciable velocity broadening, VII Zw 31 is not a Seyfert galaxy. The weighted mean topocentric redshift is $c z=16260 \pm 70 \mathrm{~km} \mathrm{~s}^{-1}$, with the wavelength calibration errors dominating the error budget. This is in excellent agreement with the redshifts measured from the $\mathrm{CO}$ emission. Table I lists the relevant measurements for the strong emission lines. Note that because of the nonphotometric quality of the night, the absolute flux calibration is uncertain by at least $50 \%$, but the relative line fluxes should be unaffected.

We can use the observed Balmer decrement ( $\mathrm{H} \alpha / \mathrm{H} \beta=8.27 \pm 0.57$ ) to estimate the internal extinction in the observed region. Assuming the foreground Galactic extinction $E_{B-V}=0.09$, and the extinction curve as parametrized by Seaton (1979), the corrected decrement is $\mathrm{H} \alpha / \mathrm{H} \beta=7.53 \pm 0.52$. Assuming that the intrinsic value is 2.85 , appropriate for an extragalactic $\mathrm{H}$ II region (cf. Veilleux and Osterbrock 1987), and using the rest-frame wavelengths and the Seaton curve, we derive the effective internal extinction $E_{B-V}=0.89 \pm 0.07$ in the central region of VII Zw 31 . The geometry of the ionized gas and dust must be fairly complex, and this number should be regarded merely as an indicative value. We then corrected the observed spectrum for the Galactic extinction, and then the rest-frame spectrum for the internal extinction. The corrected spectra are shown in Fig. 6 (for the red channel) and Fig. 7 (for the blue channel), and the corrected line energies are listed in Table I.

If we assume this reddening for the center of the galaxy, and a much smaller effect for the outskirts, the corrected color gradients would be inverted from the observed ones: the center would be much bluer than the envelope, with a color $(g-r)_{0}<0$, indicative of an early-type stellar population.

We used the extinction-corrected line ratios and the diagnostic diagrams presented by Veilleux and Osterbrock (1987) to establish the probable nature of the object. In all three line ratio diagrams, the object is clearly in the starburst/H II region domain, rather than in the AGN domain. Extinction corrections and any possible flux calibration problems have very little effect on this result because of the clever choice of line ratios by Veilleux and Osterbrock (1987). Using the line ratios and the $\mathrm{H}$ II region photoionization model by Evans and Dopita (1985), we estimate the effective ionizing temperature $T_{*} \sim 40000 \mathrm{~K}$. Keeping in mind the uncertainties of geometry and extinction, we conclude that the line emission from VII Zw 31 is powered by OB stars, rather than by an active nucleus.

One discrepant line is [O II] 3727, which appears too strong relative to the other lines if photoionization is the only mechanism operating. This is also the only emission line

TABLE I. Emission lines detected in VII Zw 31

\begin{tabular}{lccccc}
\hline \hline & & & \multicolumn{3}{c}{ Flux $\left(10^{-15} \mathrm{erg} \mathrm{cm}^{-2} \mathrm{~s}^{-1}\right)$} \\
Line & $\lambda_{\text {obs }}(\mathrm{A})$ & $c z\left(\mathrm{~km} \mathrm{~s}^{-1}\right)$ & $W_{\lambda}(\mathrm{A})$ & Observed & Corrected \\
\hline [O II] 3727 & 3926.5 & 16,050 & $30.0 \pm 1.2$ & $10.3 \pm 0.2$ & $659 \pm 13$ \\
$H \beta$ 4861 & 5124.7 & 16,244 & $10.1 \pm 0.5$ & $8.1 \pm 0.5$ & $220 \pm 14$ \\
[O III] 4959 & 5227.1 & 16,215 & $2.4 \pm 0.6$ & $1.85 \pm 0.4$ & $47 \pm 10$ \\
[O III] 5007 & 5278.8 & 16,281 & $5.6 \pm 0.4$ & $4.4 \pm 0.3$ & $107 \pm 7$ \\
[O III] 6300 & 6642.2 & 16,269 & $3.6 \pm 0.2$ & $2.85 \pm 0.1$ & $30 \pm 1$ \\
$H \alpha 6563$ & 6918.9 & 16,268 & $86.0 \pm 3.0$ & $67.0 \pm 0.5$ & $627 \pm 5$ \\
[N II] 6583 & 6940.4 & 16,256 & $35.9 \pm 0.5$ & $30.3 \pm 0.5$ & $281 \pm 5$ \\
[S II] 6716 & 7080.9 & 16,270 & $14.4 \pm 0.3$ & $10.4 \pm 0.5$ & $91 \pm 4$ \\
[S II] 6731 & 7096.0 & 16,267 & $10.9 \pm 0.2$ & $9.95 \pm 0.1$ & $87 \pm 1$ \\
\hline \hline
\end{tabular}



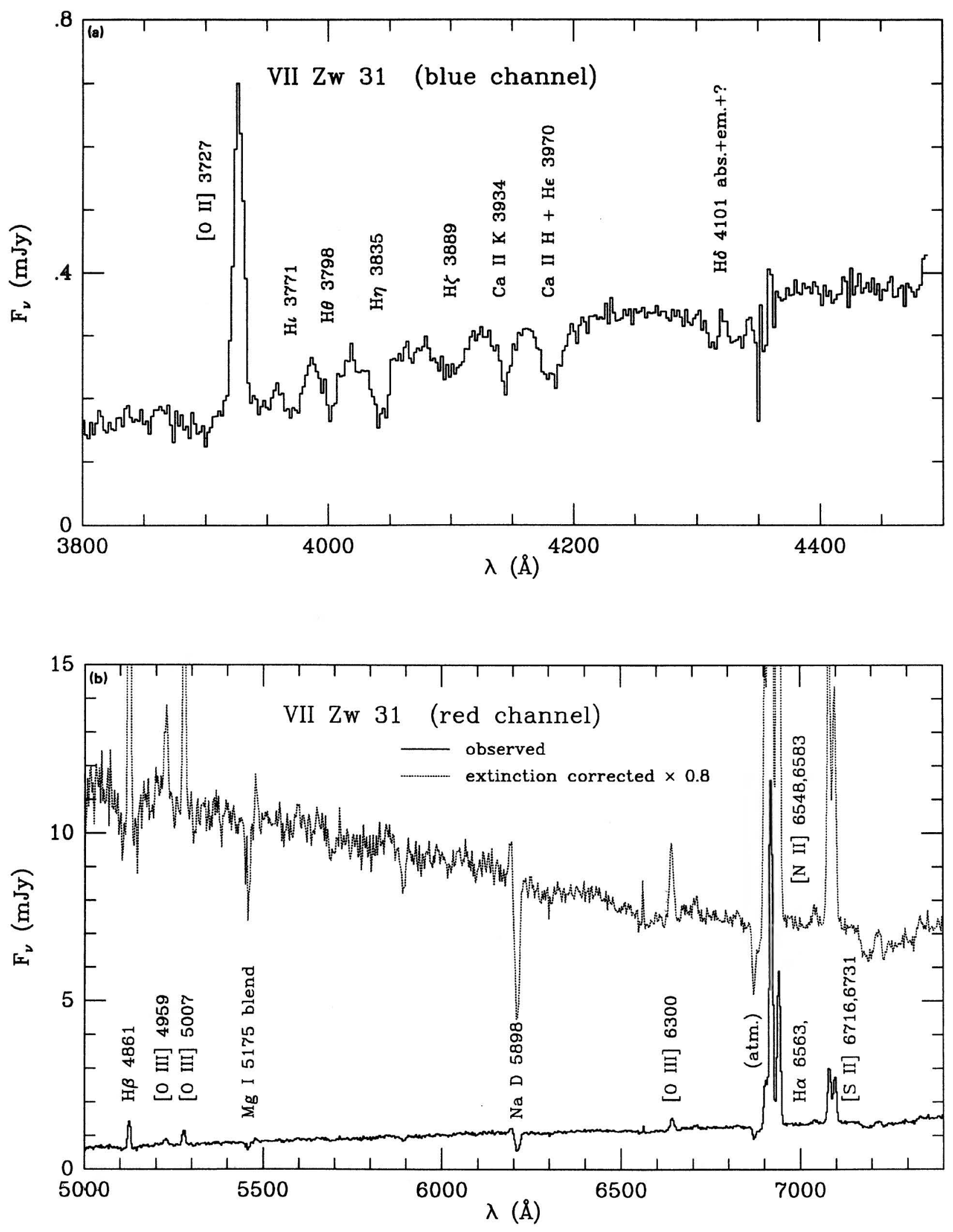

Fig. 6. The fully reduced spectra, with the emission and absorption features marked. (a) The blue channel. Note the prominent Balmer absorption lines, characteristic of a young stellar population. (b) The red channel. The dotted line shows the spectrum corrected for the estimated reddening. Note the dramatic change in the slope. The $\mathrm{H} \beta$ emission appears to fill in an absorption feature. 


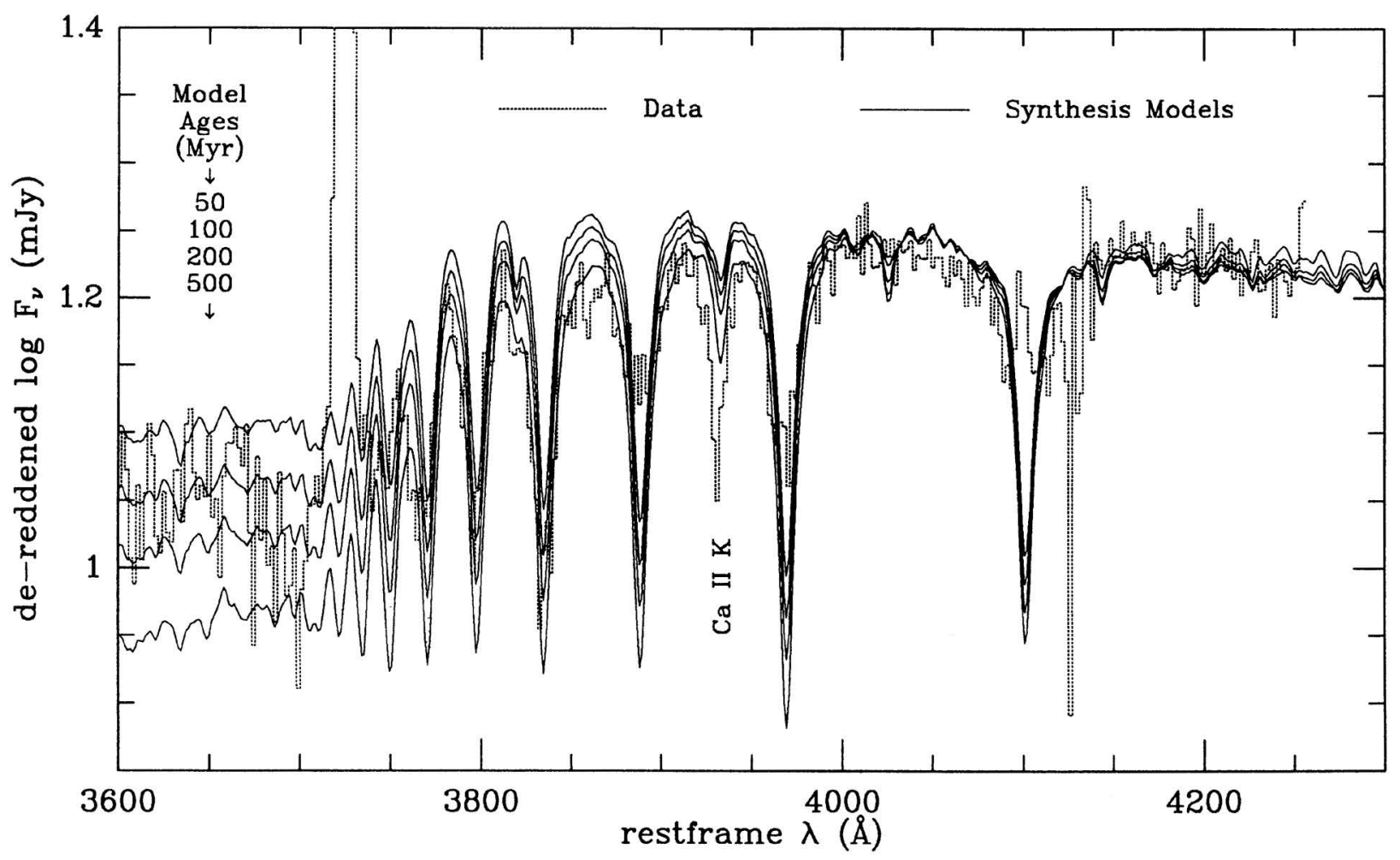

FiG. 7. Reddening-corrected blue channel spectrum, plotted against the rest-frame wavelength (dotted line), with several Bruzual population synthesis models overlaid (solid lines). The model spectra were arbitrarily shifted to coincidence at $4300 \AA$, and then jointly shifted as to match the overall continuum in the range $\sim 4000-4300 \AA$. The model ages are indicated on the right (the reddest models are the oldest). The $H \delta$ emission appears to fill in an absorption feature. Note also that the models do not reproduce the depth of the $\mathrm{Ca} I \mathrm{~K}$ line, indicating the presence of an older underlying population.

which was clearly extended outside the immediate nuclear region on our long-slit CCD spectra, and it shows a net velocity shift of $\sim 200 \mathrm{~km} \mathrm{~s}^{-1}$ with respect to the other emission lines. On the [O III] 6300/[O III] 5007 vs [O II] 3727/[O III] 5007 diagnostic diagram by Baldwin et al. (1981), the galaxy is in the region occupied by shock-heated objects. We conclude that some fraction of the [O II] 3727 emission is due to shock ionization. That, too, is consistent with the merger picture.

Finally, we can use the [S II] 6716,6731 doublet ratio to estimate the density in the observed region. From the peak intensities, we derive the ratio of 1.18 ; from the equivalent widths, 1.32; and from the line energies, 1.05; we adopt $1.2 \pm 0.1$ as the average estimate. Using the curve for the $\mathrm{H}$ il regions presented by Osterbrock (1989), we derive for the electron density:

$$
\log \left(N_{e} \sqrt{\frac{10^{4} \mathrm{~K}}{T}}\right)=2.45 \pm 0.3
$$

Assuming our estimate for $T_{*} \sim 40000 \mathrm{~K}$ we obtained $N_{e}$ $\simeq 560 \times 2^{ \pm 1}$, which is a reasonable value for a starburst region.

\section{DISCUSSION AND CONCLUSIONS}

The disturbed optical morphology of VII Zw 31 is suggestive of a recent merger. A prominent dust lane is coincident with the compact central $\mathrm{CO}$ source, and apparently is the cause of a strong color gradient observed near the center. The spectrum of the galaxy and the derived physical parameters are typical of a starburst powered by young OB stars. We thus conclude that, like many other ultraluminous IRAS galaxies, VII Zw 31 is a merger-induced starburst. We see no spectroscopic or morphological evidence for an active nucleus in this object.

This still leaves open the question of the possible protogalactic (or perhaps protodisk) nature of this object, and specifically, its age. In Fig. 7, we compare the dereddened blue spectra with the population synthesis models by Bruzual (1983, 1989). The model spectra were computed for a constant star-formation rate and with the standard Scalo (1986) IMF. The possible range of model parameters is too large and our data are of an insufficient quality to do an extensive population modeling. To wit, the IMF in this starburst may be dwarf-deficient, the star-formation rate highly nonuniform, the metallicity effects may be important, an old underlying population may be present, etc. Rather, this comparison is simply meant to be illustrative, and to give at least a rough estimate of the age of the starburst. The UV/ blue region of the spectrum is dominated by the youngest population component, and the most massive stars present. We see that the correspondence is reasonably good for the model ages $\sim 100 \mathrm{Myr}$ with an uncertainty of at least a factor of 2 ; however, the $0.5 \mathrm{Gyr}$ old model fails to reproduce the UV flux. This timescale is typical for a starburst nucleus, and is comparable to the orbital and the galaxy free-fall timescales. 
The models which fit the UV/blue part of the spectrum do not reproduce well the shape of the red continuum (there is an excess of observed red/IR light). This is also supported by our color profiles. As we already noted, absorption lines in the spectrum indicate presence of both young and old stars. The young models reproduce the strength of the Balmer absorption lines, but fail to account for the strength of the metallic lines ( $\mathrm{Ca}$ II $\mathrm{K}, \mathrm{Na} \mathrm{D}, \mathrm{Mg}$ ); these must come from an older stellar population. We thus conclude that VII $\mathrm{Zw} 31$ contains a young stellar population, with an age $\sim 10^{8}$ $\mathrm{yr}$, undoubtedly produced by the ongoing starburst, atop of an older stellar population. We are unable at this point to estimate their relative fractions by mass.

Thus, the object can be interpreted as an older galaxy, rejuvenated by a merger-induced starburst. This was already suggested as a possible explanation by Sage and Solomon (1987). If the merger interpretation is correct, the galaxy is unlikely to have a sizable disk, protogalactic or otherwise. However, the progenitors of the system must have been very gas-rich: recall that the radio observations indicate that a large fraction of the total mass is in the form of the molecular gas. Spectacular as this starburst may now be, its brightest days are probably yet to come. We propose that VII Zw 31 is a possible protostarburst galaxy, caught near the beginning of a massive star-formation episode in its life

Since a large fraction of the total mass of this system is still in the gaseous form, and ready to burn, this process may be similar to the early starbursts in at least some protogalaxies at large redshifts. VII $Z_{w} 31$ and similar objects can then be used to model some aspects of galaxy formation and its consequences, perhaps in the manner used by Djorgovski and Weir (1990).

Modern theories of the formation and evolution of galaxies and large-scale structure maintain that galaxy formation is an extended process, continuing perhaps into the present epoch. The basic mode of galaxy formation would be mergers, and starbursts introduced by them (e.g., Silk and Szalay 1987; Baron and White 1987; etc.) Vázquez and Scalo (1989; see also Scalo and Vázquez 1989) proposed a model in which mergers or the initial collapse of protogalaxies can lead to starbursts, but only after a long gestation period. VII Zw 31 may be an example of such a system, just igniting now. Some known dwarf galaxies at low redshifts, most notably I Zw 18 (Kunth and Sargent 1986; Lequeux and Viallefond 1980) may indeed be just undergoing their first burst of star formation. One candidate for a late-forming galaxy in the Virgo Cluster was recently discovered by Giovanelli and Haynes (1989). Another unusual object, Malin-1, is probably an underdeveloped large disk at a low redshift (Bothun et al. 1987; Impey and Bothun 1989). VII $\mathrm{Zw} 31$ may be another example of a late-forming galaxy. Such objects are rare and important, and may teach us about the mechanisms of galaxy formation at any redshift.

We would like to thank the staff of Palomar Observatory for their help during our observing runs, and in particular to Juan Carrasco, Dave Tennant, John Henning, and Skip Staples, and to Helen Johnston for assistance during the observations. We also thank Dr. Gustavo Bruzual for providing us with the latest version of his population synthesis code. We thank Nick Weir for his help in data reductions, and the careful reading of the manuscript. This work was supported in part by the Alfred P. Sloan Foundation and by California Institute of Technology (S.D.), and by a Caltech graduate fellowship (D.J.T.) R.R.dC. is on leave of absence from Observatorio Nacional, CNPq, Rio de Janeiro, Brazil.

\section{REFERENCES}

Baldwin, J., Phillips, M., and Terlevich, R. (1981). Publ. Astron. Soc. Pac. 93, 5.

Baron, E., and White, S. D. M. (1987). Astrophys. J. 322, 585.

Bothun, G., Impey, C., Malin, D., and Mould, J. R. (1987). Astron. J. 94 , 23.

Bruzual, G. (1983). Astrophys. J. 273, 105.

Bruzual, G. (1989). Private communication.

Burstein, D., and Heiles, C. (1982). Astron. J. 87, 1165.

Djorgovski, S. (1985). Ph. D. thesis, University of California, Berkeley.

Djorgovski, S. (1986). In Instrumentation in Astronomy, Proc. SPIE 627, 674.

Ebneter, K., Djorgovski, S., and Davis, M. (1987). Astron. J. 95, 422. Evans, I. N., and Dopita, M. A. (1985). Astrophys. J. Suppl. 58, 125. Fairclough, J. H. (1986). Mon. Not. R. Astron. Soc. 219, 1p. Giovanelli, R., and Haynes, M. (1989). Astrophys. J. Lett. (in press). Goldsmith, P., and Young, J. (1989). Astrophys. J. 341, 718. Impey, C., and Bothun, G. (1989). Astrophys J. 341, 89.

Kent, S. (1985). Publ. Astron. Soc. Pac. 97, 165.
Kunth, D., and Sargent, W. L. W. (1986). Astrophys. J. 300, 496. Lequeux, J., and Viallefond, F. (1980). Astron. Astrophys. 91, 269. Oke, J. B., and Gunn, J. E. (1982). Publ. Astron. Soc. Pac. 94, 586. Oke, J. B., and Gunn, J. E. (1983). Astrophys. J. 266, 713.

Osterbrock, D. E. (1989). Astrophysics of Gaseous Nebulae and Active Galactic Nuclei (University Science Book, Mill Valley, CA).

Sage, L., and Solomon, P. (1987). Astrophys. J. Lett. 321, L103.

Scalo, J. (1986). Fund. Cosmic Phys. 11, 1

Scalo, J., and Vázquez, E. (1989). Astrophys. Space Sci. 156, 275.

Scoville, N., Sanders, D., Sargent, A., Soifer, T., and Tinney C. (1989). Astrophys. J. Lett. 345, L25.

Seaton, M. J. (1979). Mon. Not. R. Astron. Soc. 187, 73p.

Silk, J., and Szalay, A. (1987). Astrophys. J. Lett. 323, L107.

Vázquez, E., and Scalo, J. (1989). Astrophys J. 343, 644.

Veilleux, S., and Osterbrock, D. E. (1987). Astrophys. J. Suppl. 63, 295.

Zwicky, F. (1971). Catalogue of Selected Compact Galaxies and of PostEruptive Galaxies. (Guemlingen, Switzerland). 